\title{
Modelagem e Predição de Reprovação de Acadêmicos de Cursos de Educação a Distância a partir da Conta- gem de Interações
}

\section{Modelling and Prediction of Distance Learning Students Failure by using the Count of Interactions}

\author{
Douglas Detoni \\ CDTec - Universidade Federal de Pelotas \\ Rua Gomes Carneiro, 1 - Pelotas - RS - Brasil \\ douglasdetoni92@gmail.com \\ Cristian Cechinel \\ FaE - Universidade Federal de Pelotas \\ Rua Benjamin Constant, 897 - Pelotas - RS - Brasil \\ contato@cristiancechinel.pro.br
}

\author{
Ricardo Matsumura Araújo \\ CDTec - Universidade Federal de Pelotas \\ Rua Gomes Carneiro, 1 - Pelotas - RS - Brasil \\ ricardo@inf.ufpel.edu.br
}
Resumo As altas taxas de reprovação e evasão de estudantes são frequentemente apontadas como dois dos principais obstáculos enfrentados na implementação e consolidação de cursos a Distância. A identificação com antecedência de estudantes em risco pode auxiliar de maneira decisiva o trabalho de professores e tutores. O presente trabalho descreve resultados da aplicação de téc- nicas de aprendizado de máquina nesta tarefa, utilizando como atributos unicamente contagens de interações. Os experimentos realizados demonstraram a viabilidade de utilizar apenas a quan- tidade de interações dos alunos para gerar predições razoavelmente precisas e que a introdução de atributos derivados das contagens (e.g. médias) é útil para previsões mais precisas quando a quantidade de dados é esparsa. Ainda, demonstrou-se também que as redes bayesianas se mos- traram o modelo mais adequado entre os modelos testados e que é possível treinar o modelo utilizando diferentes fontes de exemplos, como entre turmas e entre semestres diferentes. A abor- dagem apresentada aqui é aplicável virtualmente a qualquer situação onde é possível contar in- terações de qualquer tipo.

Palavras-Chave: Aprendizado de máquina, Predição de reprovação, Educação a Distância, Mineração de Dados Educacionais

\begin{abstract}
The high students' failure and dropout rates are often pointed as two of the main obstacles for the implementation and consolidation of Distance Learning courses. The early identification of atrisk students can decisively aid teachers and assistants. The present work describes results of the application of machine learning techniques to perform this task using only the count of interactions as attributes. The experiments demonstrated the feasibility of using only the counts of students interaction to generate reasonably accurate predictions, and that introducing attributes derived from the counts (e.g. means) is useful for more accurate predictions when data is sparse. Moreover, it was also demonstrated that Bayesian networks are adequate for the problem and that it is possible to train models in different sources of examples, such as between different groups and semesters. The present approach is virtually applicable in any scenario where it is possible to count interactions of any kind.
\end{abstract}

Keywords: Machine learning, Predicting at-risk students, Distance Learning, Educational Data Mining 


\section{Introdução}

A Educação a Distância (EAD) no Brasil tem se consolidado com diversos estudantes optando por essa modalidade de ensino para ampliar suas formações e realização profissional. Em 2012, segundo dados do Censo EAD 2013 [1], foram ofertados 9.376 cursos. Um total de 5.772 .466 matrículas foram registradas, com um 52,5\% de aumento em relação a 2011. Já o número de conclusões, em 2012, foi de 1.589.374. Esses dados mostram a evolução da Educação a Distância e como ela está se tornando uma ferramenta muito importante na formação dos cidadãos brasileiros. Contudo, a EAD ainda enfrenta alguns obstáculos a serem ultrapassados. Resistência de educandos e educadores, desafios organizacionais e custos de produção são alguns deles mas, sem dúvida, um dos maiores obstáculos é a evasão de alunos dos cursos e instituições. De acordo com o Censo EAD 2013 [1], a evasão em cursos a distância autorizados pelo Ministério da Educação chegou a atingir um pico de $20,5 \%$ em 2011, tendo sido de $18,6 \%$ em 2010 e de $11,74 \%$ em 2012.

Um dos principais diferenciais de cursos EAD é a grande quantidade de dados gerada pelas interações no ambiente educacional, o que abre novas possibilidades para estudar e compreender estas interações [2] [3]. Algumas áreas de pesquisas surgiram nos últimos anos com intuito de auxiliar em questões como essas. A Mineração de Dados Educacionais (do inglês Educational Data Mining EDM) é uma área de pesquisa interdisciplinar que lida com o desenvolvimento de métodos para explorar dados originados no contexto educacional [4]. Juntamente com a EDM temos a Analítica de Aprendizagem (do inglês Learning Analytics - LA) que busca medir, coletar, analisar e reportar dados sobre estudantes e seus contextos, com o propósito de entender e otimizar seu aprendizado e o ambiente onde ele ocorre [5]. As interações dos alunos e professores com os ambientes virtuais de aprendizagem (AVA) proveem os dados que alimentam as pesquisas nessas áreas e possibilitam a descoberta de novos conhecimentos.

Um dos grandes desafios dos pesquisadores é desenvolver métodos capazes de prever o comportamento dos estudantes, de modo a possibilitar a intervenção de professores/tutores, ou demais envolvidos, visando resgatar o estudante antes que ele reprove [6]. A possibilidade de identificação de estudantes com alto risco de evasão e reprovação a partir de modelos automáticos foi apontada por Baker et al. [7] como um dos potenciais problemas a serem atacados pela comunidade brasileira que atua na área de mineração de dados educacionais. Outros objetivos importantes da área também são, por exemplo, o de detectar estudantes que precisam de ajuda, classificar diferentes grupos de estudantes, e detectar trapaças no sistema [4]. Segundo Romero and Ventura [4], existe a necessidade de desenvolver ferramentas específicas e fáceis de usar, para que professores/tutores não familiarizados com as técnicas de EDM e LA também possam se valer das descobertas dessas áreas. É importante também integrar as ferramentas a AVAs tradicionalmente já usados. Outro aspecto a ser trabalhado é a padronização dos dados e modelos, pois muitas ferramentas possuem padrões próprios e específi$\cos$, e que não podem ser integrados por outras ferramentas.

O principal objetivo deste trabalho é desenvolver e testar técnicas e modelos de aprendizado de máquina para predição da reprovação de alunos de educação a distância utilizando o registro de interações dentro do ambiente Moodle entre alunos, tutores e professores em cursos da Universidade Federal de Pelotas.

Adicionalmente, é nosso objetivo analisar unicamente atributos agregados - i.e. números totais de interações, sem especificar o tipo de atividade sendo realizada pelo estudante. Este objetivo é motivado pela heterogeneidade de atividades entre cursos e plataformas; ao utilizar como atributo atividades específicas (e.g. fórum, chat), acaba-se por ancorar a técnica aos cursos específicos sendo analisados, tornando-a pouco generalizável.

Este trabalho é uma extensão de um trabalho anteriormente publicado no SBIE 2014 [8] e está estruturado da seguinte maneira. A seção 2 descreve alguns trabalhos relacionados com a predição da situação de acadêmicos em AVAs que se aproximam do presente trabalho. A seção 3 apresenta o contexto de coleta dos dados e o pré-processamento realizado. Na seção 4 são descritos as análises realizadas e na seção 5 os resultados encontrados. As considerações finais e propostas de trabalhos futuros são apresentadas na seção 6 .

\section{Trabalhos Relacionados}

A noção de interação dentro de AVA é mencionada em diversos trabalhos relacionados a $\mathrm{EAD}$, e já está relativamente bem estabelecido na literatura que o sucesso dos estudantes de cursos a distância está correlacionado com o seu engajamento dentro dos AVA [9] [10] [11] [12] [13]. A eficácia e a eficiência de estudantes têm frequentemente sido associadas a diferentes medidas de suas interações dentro dos AVA, e normalmente possuem uma alta correlação com o sucesso dos mesmos nos cursos. Por exemplo, Murray et al. [14] observaram que estudantes que apresentaram as mais altas taxas de acesso aos conteúdos no AVA de um curso a distância, também receberam as melhores notas, e Dickson [15] descobriu que o número total de cliques dados por estudantes é fortemente correlacionado com as suas notas finais em um curso. Garrison et al. [16] projetaram um framework conceitual identificando os ele- 
mentos críticos necessários para uma experiência educacional mediada por computador obter sucesso. De acordo com os autores, os elementos centrais são a presença cognitiva (até onde cada um é capaz de construir significações), a presença social (a habilidade do estudante de se projetar para os outros participantes de uma comunidade virtual como uma pessoa real) e a presença pedagógica (projeto de um experiência educacional que facilite e melhore as presenças cognitiva e social para que atinja objetivos de aprendizagem). De acordo com Swan [17], cada um desses elementos pode ser equiparado a um modo diferente de interatividade que pode afetar diretamente a eficiência da aprendizagem, por exemplo, as interações com o curso se equivaleriam com a presença cognitiva, as interações entre pares se equivaleriam com a presença social, e as interações com professores e tutores se equivaleriam a presença pedagógica.

Considerando que a EAD permite a medição do engajamento dos estudantes a partir da observação dos logs do sistema e da avaliação da intensidade de suas interações nas diferentes atividades das salas virtuais [18], diversos trabalhos na literatura tem focado seus esforços na predição automática do desempenho acadêmico a partir da utilização desses dados. Por exemplo, Manhães et al. [19] utilizaram técnicas de mineração de dados para prever a evasão de estudantes em cursos presenciais da Escola Politécnica da Universidade do Rio de Janeiro. Dez modelos diferentes foram testados, com acurácia média variando entre $75 \%$ e $80 \%$, com Perceptron de Múltiplas Camadas e Florestas Aleatórias apresentando os melhores desempenhos. Ainda, Gottardo et al. [20], apresentam resultados de tentativas de prever a avaliação de desempenho de alunos de educação a distância. Os autores relatam taxas de precisão acima de $74 \%$, com o uso de uma grande variedade de atributos. O presente trabalho diferencia-se ao tentar prever a reprovação de alunos e não seus desempenhos específicos.

Rodrigues et al. [21] apresentaram um estudo da viabilidade do uso de um modelo de regressão linear para também prever o desempenho dos alunos. Este trabalho também utilizou dados do ambiente virtual de aprendizagem Moodle, além da utilização de séries temporais. Diversos atributos foram utilizados, incluindo a quantidade de interações ao longo das semanas em fóruns, vídeos, materiais de apoio, entre outros.

Por último, Gottardo et al. [22] realizam a tarefa de predição do desempenho do aluno utilizando Sistemas de Recomendação e Acoplamento de Classificadores. Os dados são relativos a um curso com 252 alunos. Os dados para treinamento dos classificadores foram extraídos da tabela de logs e separados por tipos, como por exemplo: assignment, fóruns, uploads, entre outros. Cada tipo transformou-se em um atributo, juntamente com a situação acadê- mica do aluno. Os classificadores C4.5 e Naive Bayes foram utilizados no trabalho. O melhor resultado foi aproximadamente $80 \%$ de predição, utilizando $40 \%$ do conjunto de dados, com o classificador Naive Bayes.

Como comentado na introdução, o presente trabalho utiliza a contagem das interações dos estudantes para a predição de sua situação sem discriminar qual o tipo dessa interação (visualização de atividade, chat, fórum, etc). A próxima seção descreve o contexto de coleta dos dados, o seu pré-processamento e os atributos testados nos modelos.

\section{Descrição dos Dados}

\subsection{Contexto dos cursos}

A Universidade Federal de Pelotas (UFPEL) oferece cursos de graduação, pós-graduação e extensão na modalidade a distância. São mais de 4000 alunos matriculados e espalhados por diversos polos do Rio Grande do Sul. Para a realização do trabalho, foram obtidos os dados anonimizados dos cursos a distância de Licenciatura em Educação do Campo (CLEC) e de Licenciatura em Pedagogia (CLPD). É importante notar que estes cursos seguem um modelo de execução das disciplinas que difere do modelo presencial convencional. Normalmente, um curso é dividido em semestres, onde diversas disciplinas são ministradas paralelamente ao longo do mesmo. No caso do CLEC e do CLPD, as disciplinas são ministradas de maneira sequencial (com no máximo 2 disciplinas acontecendo em paralelo). Normalmente são ministradas em torno de 5 disciplinas por semestre com duração média de cinco a sete semanas.

Foram utilizadas disciplinas do primeiro e segundo semestre dos cursos citados. Ambos os semestres ocorreram no ano de 2013, totalizando 329 estudantes únicos. Contabilizando os 4 semestres utilizados, temos um total de 604 alunos, 72 tutores e 80 professores. A Tabela 1 apresenta o número de alunos para cada situação.

Tabela 1. Total de alunos em cada curso e semestre

\begin{tabular}{|l|l|l|l|l|}
\hline Curso & Semestre & Alunos & Aprovados & $\begin{array}{l}\text { Reprova- } \\
\text { dos }\end{array}$ \\
\hline $\begin{array}{l}\text { Educação } \\
\text { do Campo } \\
\text { (CLEC) }\end{array}$ & $1^{\circ}$ & 133 & 103 & 30 \\
\cline { 2 - 5 } & $2^{\circ}$ & 94 & 88 & 6 \\
\hline \multirow{2}{*}{$\begin{array}{l}\text { Pedagogia } \\
\text { (CLPD) }\end{array}$} & $1^{\circ}$ & 196 & 137 & 59 \\
\cline { 2 - 5 } & $2^{\circ}$ & 172 & 119 & 53 \\
\hline
\end{tabular}

\subsection{Coleta e Pré-processamento dos Dados}

Os dados foram extraídos dos registros de acesso dos 
cursos fornecidos pela plataforma Moodle. Destes registros, para cada interação de cada usuário, extraiu-se apenas o identificador do usuário, sua função (professor, tutor ou aluno) e a data e hora da interação. Assim, ao contrário de trabalhos anteriores, procuramos utilizar apenas o número total de interações dos estudantes, tutores e professores com o sistema, sem detalhamento do tipo de atividade sendo realizada. A motivação por trás desta decisão é a possibilidade de permitir que a metodologia proposta seja generalizável a diferentes cursos, plataformas e execuções, sem gerar uma dependência em atributos excessivamente específicos. A Tabela 2 apresenta as quantidades totais de interações por curso em cada semestre, além da média de interações por disciplina. No total foram extraídas cerca de 835 mil interações.

Tabela 2. Total de interações por curso e semestre e média de interações por disciplina

\begin{tabular}{|l|l|l|l|}
\hline $\begin{array}{l}\text { Nome do } \\
\text { Curso }\end{array}$ & Semestre & $\begin{array}{l}\text { Total de Inte- } \\
\text { rações }\end{array}$ & $\begin{array}{l}\text { Média de Intera- } \\
\text { cões por Disci- } \\
\text { plina }\end{array}$ \\
\hline $\begin{array}{l}\text { Educação do } \\
\text { Campo }\end{array}$ & $1^{\circ}$ & 230.164 & $46.032,8$ \\
\cline { 2 - 4 } & $2^{\circ}$ & 169.453 & $33.890,6$ \\
\hline \multirow{2}{*}{ Pedagogia } & $1^{\circ}$ & 290.552 & $58.110,4$ \\
\cline { 2 - 4 } & $2^{\circ}$ & 144.666 & $29.533,2$ \\
\hline
\end{tabular}

Cada aluno foi rotulado como aprovado ou reprovado em cada disciplina de acordo com a situação informada nos registros acadêmicos. Cada disciplina possui duração de 7 semanas e as interações de cada aluno foram agrupadas de forma semanal. Assim, cada aluno em uma disciplina é descrito por um vetor de 7 atributos indicando o número total de interações daquele aluno em cada semana.

Considerando que o objetivo principal é predizer a situação final do aluno com a maior antecedência possível dentro da disciplina, foram gerados diversos conjuntos de dados com base nas combinações incrementais das sete semanas de interações existentes para a disciplina. Assim, foram gerados conjuntos de dados contendo somente a primeira semana de interações, contendo a primeira e a segunda semana, e assim por diante, até ser gerado o conjunto de dados com todas as 7 semanas de interações. Um conjunto de dados consiste em todos os exemplos de alunos de um dado semestre de um curso.

\subsection{Atributos Derivados}

Ainda que a série temporal gerada pelo número absoluto de interações dos alunos possa ser útil, geramos também um conjunto de atributos derivados que podem auxiliar na contextualização desses valores. $O$ conjunto total de atributos utilizados e a descrição dos mesmos são apresen- tados na Tabela 3. Todos atributos são derivados unicamente na contagem total de interações e relações entre interações de alunos, tutores e professores.

Este conjunto de atributos foi escolhido de modo que certos aspectos dos dados originais fossem enfatizados. Esse é o caso da média, mediana e número de semanas com zero interações. Os demais foram criados com base em hipóteses, tentando revelar características ainda não conhecidas ou relações entre os dados de professores e tutores com os alunos. A principal hipótese é de que o número absoluto de interações dos estudantes tem pouco valor, sendo necessário colocá-lo no contexto do número de interações da turma como um todo e dos tutores e professores. Também foi buscada uma inter-relação entre os dados de um exemplo de aluno com os dados dos demais exemplos de aluno da disciplina, caso este do fator de empenho.

Tabela 3. Conjunto de Atributos

\begin{tabular}{|l|l|}
\hline Atributo & Descrição \\
\hline Situação Acadêmica & $\begin{array}{l}\text { Situação final do aluno na disciplina } \\
\text { (rótulo do exemplo) }\end{array}$ \\
\hline $\begin{array}{l}\text { Interações por Semana } \\
\text { (1 até 7 semanas) }\end{array}$ & Número de interações na semana. \\
\hline Média & $\begin{array}{l}\text { Média do total de interações pelo nú- } \\
\text { mero de semanas. }\end{array}$ \\
\hline Mediana & $\begin{array}{l}\text { Mediana do conjunto de interações por } \\
\text { semana. }\end{array}$ \\
\hline Semanas Zeradas & $\begin{array}{l}\text { Número de semanas com zero intera- } \\
\text { ços. }\end{array}$ \\
\hline Média da Diferença & $\begin{array}{l}\text { Média da diferença entre a semana } i \text { e a } \\
\text { semana } i+1\end{array}$ \\
\hline Razão com Professores & $\begin{array}{l}\text { Razão entre o total de interações do } \\
\text { aluno e dos professores. }\end{array}$ \\
\hline Razão com Tutores & $\begin{array}{l}\text { Razão entre o total de interações do } \\
\text { aluno e dos tutores. }\end{array}$ \\
\hline 1 até 7 semanas $)$ & $\begin{array}{l}\text { Razão entre as interações da semana do } \\
\text { nano e a média de interações da turma } \\
\text { naquela semana. }\end{array}$ \\
\hline
\end{tabular}

\section{Metodologia de Análise}

Neste artigo, estamos interessados em verificar a eficácia de diferentes modelos em prever a situação de alunos em diferentes situações. Em particular, focamos em dois casos: a) utilizando somente o número absoluto de interações dos estudantes e b) adicionando os atributos derivados. O primeiro caso visa identificar o potencial do modelo de dados constituído somente pela situação do aluno e as interações que ele teve em cada semana. O segundo caso 
utiliza as mesmas informações do primeiro, porém acrescentando os atributos derivados (média, mediana, média da diferença, semanas zeradas, razão com os professores, razão com os tutores e o fator de empenho de cada semana).

Para cada análise, dividiu-se os conjuntos de exemplos em um conjunto de treinamento e um conjunto de teste. Esta divisão foi feita de duas formas para testar duas situações distintas. Na primeira forma, denominada "entre turmas", a divisão é feita dentro de um mesmo semestre do mesmo curso, utilizando turmas distintas para treino e teste. Na segunda forma, denominada "entre semestres", a divisão é feita entre semestres distintos.

Para tanto, cada conjunto de dados foi dividido aleatoriamente em dois conjuntos contendo a mesma quantidade de alunos, ou seja, cada turma de alunos foi transformada em duas turmas (A e B). No primeiro formato (entre turmas) uma turma (ex: turma A) de um semestre de um curso foi utilizada para treinar o modelo, e a outra turma (ex: turma B) daquele semestre daquele curso foi utilizada para avaliar o modelo (e vice-versa). No segundo formato (entre semestres), cada uma das turmas (A e B) de cada semestre foi utilizada para treinar os modelos, e os mesmos foram avaliados com as turmas A e B do semestre seguinte (e vice-versa).

Esse processo de divisão foi repetido 5 vezes, gerando conjuntos aleatórios diferentes a cada repetição. As médias dos resultados da aplicação dos classificadores nestas repetições são reportadas.

Os seguintes modelos foram aplicados aos dados: Rede Bayesiana, Rede Neural (Perceptron de Múltiplas Camadas), C4.5 e Floresta Aleatória. Utilizou-se a implementação da biblioteca WEKA [23], com os parâmetros padrões para todos modelos.

\section{Resultados}

De modo geral, as acurácias médias de classificação alcançaram altos índices já para os modelos que utilizavam somente os dados das interações da primeira semana, tendo sido possível, por exemplo, gerar modelos com acurácia média superior a $90 \%$.

No entanto, medidas de acurácia dos classificadores não são adequadas aqui, já que as classes não são balanceadas - há muito mais candidatos retidos do que evadidos, fazendo com que classificadores que somente indicam retenção já tenham alta acurácia sem serem realmente úteis. Assim, focamos aqui principalmente na capacidade dos classificadores em indicar corretamente reprovações - isto é, a fração corretamente classificada de estudantes reprovados. A precisão na classificação de alunos aprovados manteve-se sempre alta, entre $75 \%$ e $95 \%$, demonstrando que a tarefa de prever reprovação é substancialmente mais difícil.

A seguir descreveremos os resultados dos experimentos relacionados aos dois cursos estudados.

\subsection{Classificação utilizando somente número de interações}

Como é possível observar na Tabela 4 e na Tabela 5 , os modelos se tornam mais precisos conforme mais semanas são disponibilizadas ao modelo. Quando utilizamos somente as quantidades de interações da primeira semana, as precisões para classificar alunos reprovados são próximas a zero.

Observa-se no entanto que há uma diminuição de ganhos com o passar das semanas - além da quinta semana novas semanas acrescentam muito pouco à precisão do modelo. Também é evidente que os cursos diferentes levam a precisões também distintas, com a aplicação no CLEC fornecendo resultados consistentemente piores. Parte desta diferença pode ser explicada pelo fato do CLEC possuir três vezes menos alunos reprovados (em números absolutos), o que implica menos dados para o treinamento dos modelos.

Para ambos os cursos as Redes Bayesianas obtiveram os melhores resultados para um número suficientemente grande de semanas (maior ou igual a 3). Para as semanas iniciais as Florestas Aleatórias mostraram melhores resultados em quase todos os casos.

Tabela 4. Precisões para classificação de reprovados - experimento sem utilização de atributos - Curso de Licenciatura em Educação do Campo

\begin{tabular}{|c|c|c|c|c|c|c|c|c|}
\hline \multirow{2}{*}{ Caso } & \multirow{2}{*}{ Modelo } & \multicolumn{7}{|c|}{ Semana } \\
\hline & & $\mathrm{S} 1$ & $\mathrm{~S} 2$ & S3 & $\mathrm{S} 4$ & S5 & S6 & S7 \\
\hline \multirow{3}{*}{ Entre Semestres } & Rede Bayesiana & 0,0 & 0,22 & 0,44 & 0,51 & 0,60 & 0,63 & 0,64 \\
\hline & Rede Neural & 0,0 & 0,16 & 0,33 & 0,45 & 0,49 & 0,51 & 0,51 \\
\hline & $\mathrm{C} 4.5$ & 0,0 & 0,21 & 0,37 & 0,40 & 0,52 & 0,56 & 0,56 \\
\hline
\end{tabular}




\begin{tabular}{|l|l|l|l|l|l|l|l|l|}
\hline & Floresta Aleatória & 0,1 & 0,33 & 0,36 & 0,40 & 0,48 & 0,51 & 0,54 \\
\hline \multirow{5}{*}{ Entre Turmas } & Rede Bayesiana & 0,0 & 0,24 & 0,45 & 0,51 & 0,58 & 0,62 & 0,66 \\
\cline { 2 - 10 } & Rede Neural & 0,0 & 0,14 & 0,33 & 0,46 & 0,49 & 0,53 & 0,56 \\
\cline { 2 - 10 } & C4.5 & 0,0 & 0,20 & 0,39 & 0,42 & 0,51 & 0,59 & 0,60 \\
\cline { 2 - 10 } & Floresta Aleatória & 0,1 & 0,28 & 0,33 & 0,41 & 0,50 & 0,56 & 0,61 \\
\hline
\end{tabular}

Tabela 5. Precisões para classificação de reprovados - experimento sem utilização de atributos - Curso de Licenciatura em Pedagogia

\begin{tabular}{|c|c|c|c|c|c|c|c|c|}
\hline \multirow{2}{*}{ Caso } & \multirow{2}{*}{ Modelo } & \multicolumn{7}{|c|}{ Semana } \\
\hline & & $\mathrm{S} 1$ & $\mathrm{~S} 2$ & $\mathrm{~S} 3$ & $\mathrm{~S} 4$ & S5 & S6 & S7 \\
\hline \multirow{4}{*}{ Entre Semestres } & Rede Bayesiana & 0,00 & 0,53 & 0,60 & 0,66 & 0,70 & 0,71 & 0,72 \\
\hline & Rede Neural & 0,00 & 0,12 & 0,50 & 0,55 & 0,52 & 0,55 & 0,57 \\
\hline & $\mathrm{C} 4.5$ & 0,00 & 0,50 & 0,58 & 0,58 & 0,57 & 0,58 & 0,57 \\
\hline & Floresta Aleatória & 0,01 & 0,49 & 0,54 & 0,57 & 0,57 & 0,58 & 0,59 \\
\hline \multirow{4}{*}{ Entre Turmas } & Rede Bayesiana & 0,00 & 0,46 & 0,60 & 0,67 & 0,71 & 0,73 & 0,73 \\
\hline & Rede Neural & 0,00 & 0,12 & 0,45 & 0,52 & 0,48 & 0,56 & 0,57 \\
\hline & $\mathrm{C} 4.5$ & 0,00 & 0,45 & 0,55 & 0,56 & 0,59 & 0,59 & 0,58 \\
\hline & Floresta Aleatória & 0,02 & 0,48 & 0,54 & 0,55 & 0,55 & 0,57 & 0,59 \\
\hline
\end{tabular}

\subsection{Classificação utilizando atributos deriva- dos}

Para os modelos treinados com atributos derivados temos um comportamento semelhante aos do experimento anterior. De modo geral os modelos também tornam-se mais precisos à medida que as informações de interação de cada semana vão sendo disponibilizadas e Redes Bayesianas se mostraram o modelo mais adequado novamente. Os maiores ganhos ao utilizar os atributos derivados encontram-se nas primeiras semanas, quando se utiliza Redes Bayesianas. À medida que mais semanas são disponibilizadas, a diferença decresce e, na última semana, não há diferenças significativas.

Tabela 6. Precisões para classificação de reprovados - experimento com a utilização de atributos - Curso de Licenciatura em Educação do Capo

\begin{tabular}{|c|c|c|c|c|c|c|c|c|}
\hline \multirow{2}{*}{ Testes } & \multirow{2}{*}{ Modelo } & \multicolumn{7}{|c|}{ Semana } \\
\hline & & $\mathrm{S} 1$ & $\mathrm{~S} 2$ & $\mathrm{~S} 3$ & $\mathrm{~S} 4$ & S5 & S6 & $\mathrm{S} 7$ \\
\hline \multirow{4}{*}{ Entre Semestres } & Rede Bayesiana & 0,32 & 0,48 & 0,54 & 0,54 & 0,65 & 0,66 & 0,63 \\
\hline & Rede Neural & 0,07 & 0,22 & 0,38 & 0,42 & 0,46 & 0,55 & 0,58 \\
\hline & $\mathrm{C} 4.5$ & 0,02 & 0,25 & 0,34 & 0,37 & 0,48 & 0,50 & 0,53 \\
\hline & Floresta Aleatória & 0,06 & 0,26 & 0,30 & 0,37 & 0,41 & 0,47 & 0,46 \\
\hline \multirow{4}{*}{ Entre Turmas } & Rede Bayesiana & 0,35 & 0,48 & 0,52 & 0,57 & 0,64 & 0,68 & 0,67 \\
\hline & Rede Neural & 0,08 & 0,24 & 0,41 & 0,44 & 0,49 & 0,57 & 0,59 \\
\hline & $\mathrm{C} 4.5$ & 0,05 & 0,27 & 0,36 & 0,39 & 0,49 & 0,57 & 0,64 \\
\hline & Floresta Aleatória & 0,11 & 0,27 & 0,33 & 0,41 & 0,45 & 0,54 & 0,51 \\
\hline
\end{tabular}


Tabela 7. Precisões para classificação de reprovados - experimento com utilização de atributos - Curso de Licenciatura em Pedagogia

\begin{tabular}{|c|c|c|c|c|c|c|c|c|}
\hline \multirow{2}{*}{ Testes } & \multirow{2}{*}{ Modelo } & \multicolumn{7}{|c|}{ Semana } \\
\hline & & $\mathrm{S} 1$ & $\mathrm{~S} 2$ & $\mathrm{~S} 3$ & $\mathrm{~S} 4$ & S5 & S6 & S7 \\
\hline \multirow{4}{*}{ Entre Semestres } & Rede Bayesiana & 0,44 & 0,59 & 0,68 & 0,70 & 0,70 & 0,70 & 0,72 \\
\hline & Rede Neural & 0,00 & 0,39 & 0,54 & 0,60 & 0,60 & 0,63 & 0,64 \\
\hline & $\mathrm{C} 4.5$ & 0,00 & 0,48 & 0,56 & 0,59 & 0,60 & 0,62 & 0,64 \\
\hline & Floresta Aleatória & 0,05 & 0,45 & 0,52 & 0,57 & 0,58 & 0,60 & 0,60 \\
\hline \multirow{4}{*}{ Entre Turmas } & Rede Bayesiana & 0,67 & 0,66 & 0,71 & 0,71 & 0,74 & 0,78 & 0,80 \\
\hline & Rede Neural & 0,01 & 0,44 & 0,57 & 0,59 & 0,63 & 0,71 & 0,72 \\
\hline & $\mathrm{C} 4.5$ & 0,05 & 0,49 & 0,54 & 0,56 & 0,62 & 0,69 & 0,72 \\
\hline & Floresta Aleatória & 0,13 & 0,46 & 0,52 & 0,55 & 0,58 & 0,68 & 0,69 \\
\hline
\end{tabular}

\subsection{Comparação dos resultados}

\subsubsection{Modelos de Predição para o Curso de Licenciatura em Educação do Campo}

A Figura 1 compara o desempenho obtido por semana utilizando Redes Bayesianas e as duas combinações de atributos para o Curso de Licenciatura em Educação do Campo. Observa-se que, para as semanas finais, as precisões dos modelos são virtualmente idênticas. No entanto, para semanas iniciais, a utilização dos atributos derivados torna-se substancialmente vantajosa. A partir da quarta semana os resultados se aproximam, convergindo para essencialmente a mesma precisão na última semana.

Figura 1. Comparação de desempenho a cada semana utilizando Redes Bayesianas e diferentes combinações de atributos - Curso de Licenciatura em Educação do Campo

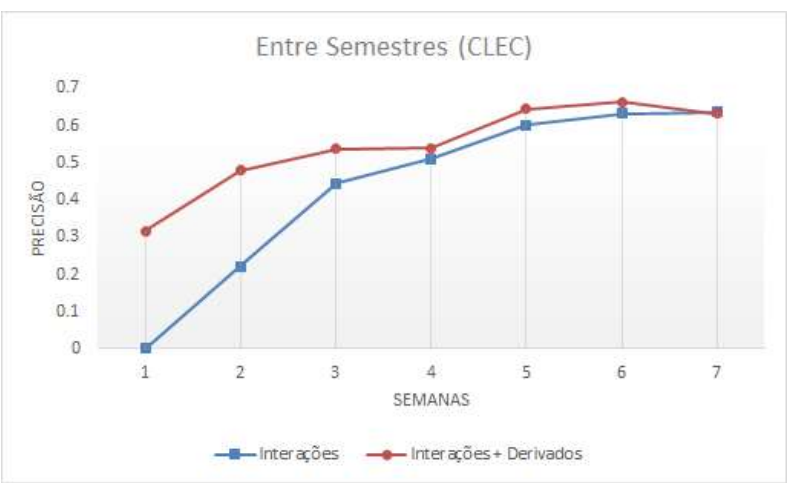

(a) Comparação entre semestres

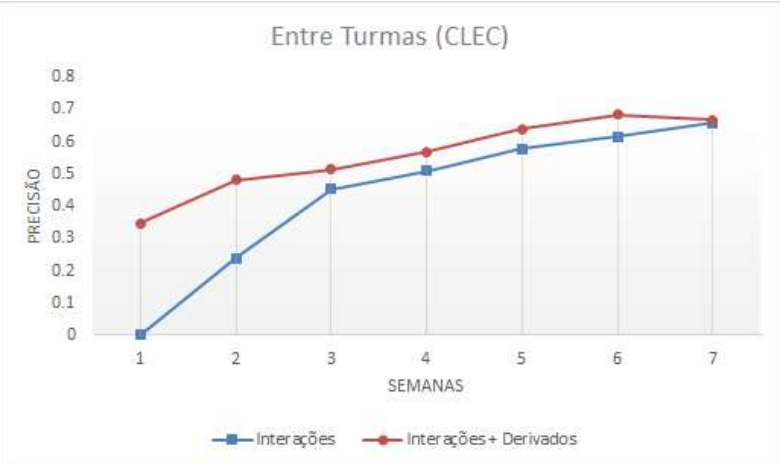

(b) Comparação entre turmas

Observa-se ainda que a precisão a cada semana é essencialmente a mesma independentemente de o modelo ser treinado entre turmas ou entre semestres (veja Figura 2). Este é um resultado interessante, pois demonstra uma invariância no resultado em relação a origem do treinamento. Isto é, um modelo pode ser treinado com dados de um semestre para ser aplicado no semestre posterior, bem como treinado em um conjunto de estudantes para ser aplicado em outros estudantes. 
Figura 2. Comparação de precisão por semana utilizando Rede Bayesiana e todos atributos disponíveis

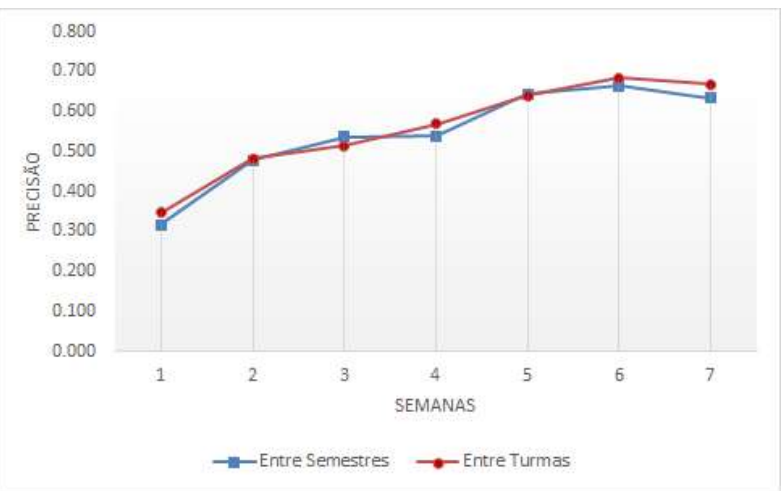

\subsubsection{Modelos de Predição para o Curso de Licenciatura em Pedagogia}

A Figura 3 apresenta a comparação ao longo das semanas entre o desempenho dos modelos de Redes Bayesianas que utilizaram somente as interações e aqueles que utilizaram os atributos derivados para o Curso de Licenciatura em Pedagogia.

De maneira similar ao que ocorre com os modelos gerados para o Curso de Licenciatura em Educação do Campo, aqui também pode-se observar que a utilização dos atributos derivados faz com que os modelos apresentem uma precisão consideravelmente melhor nas primeiras três semanas das disciplinas, sendo que a partir da quarta semana as diferenças entre os desempenhos de ambos os tipos de modelos passam a ser menos significativas.

Figura 3. Comparação de desempenho a cada semana utilizando Redes Bayesianas e diferentes combinações de atributos - Curso de Licenciatura em Pedagogia

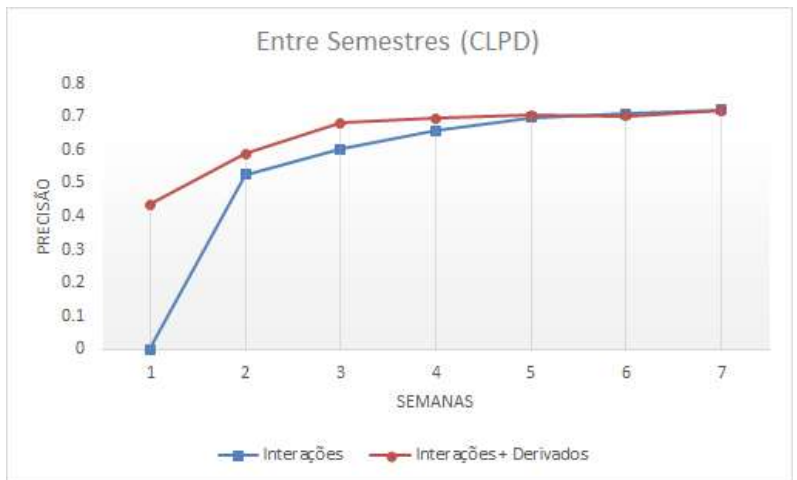

(a) Comparação entre semestres

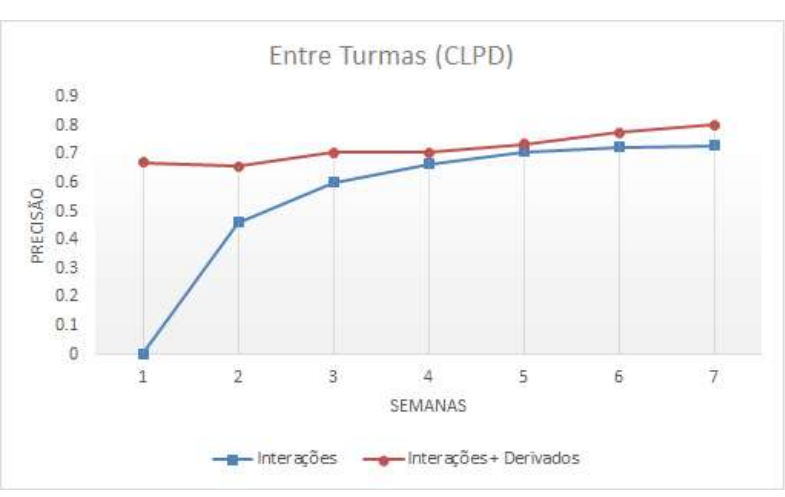

(b) Comparação entre turmas

Ainda, para o Curso de Licenciatura em Pedagogia, os modelos se comportam virtualmente diferentes dependendo da origem do treinamento (Figura 4), sendo que os modelos treinados "Entre Turmas" apresentam melhores precisões. Isso ocorre, sobretudo, na primeira, segunda, sexta e sétima semana do curso. Diferentemente dos testes realizados para o Curso de Licenciatura em Educação do Campo, aqui não é possível afirmar que os modelos gerados a partir de diferentes origens de treinamentos são invariáveis.

Figura 4. Comparação de precisão por semana utilizando Rede Bayesiana e todos atributos disponíveis - Curso de Licenciatura em Pedagogia

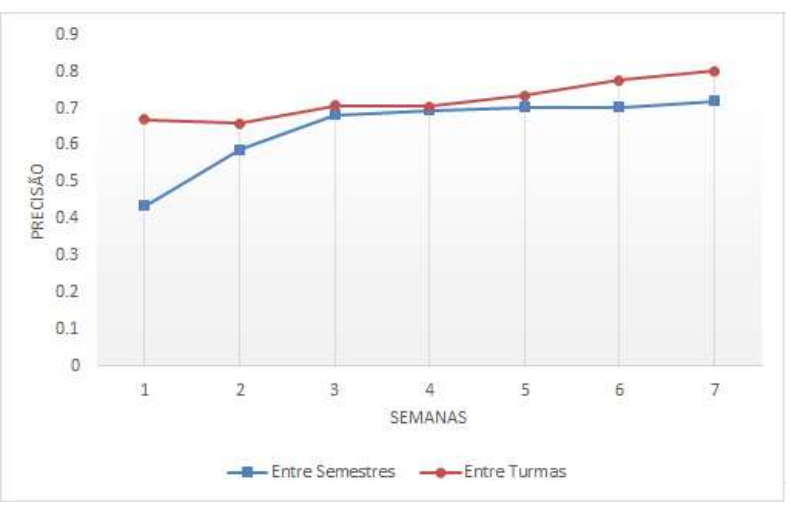

\section{Considerações Finais e Trabalhos Futuros}

A predição com antecedência de alunos em risco de repetência e evasão em cursos de educação à distância pode auxiliar de maneira decisiva o trabalho de professores e tutores que teriam a oportunidade de ajustar seus procedimentos pedagógicos de maneira personalizada para esses estudantes.

O presente trabalho descreveu resultados importantes obtidos a partir da aplicação de técnicas de aprendizado de 
máquina para o problema de predição de reprovação de estudantes em dois cursos à distância (Licenciatura em Pedagogia e Licenciatura em Educação do Campo) da Universidade Federal de Pelotas.

A principal contribuição deste trabalho está em demonstrar a viabilidade da utilização apenas da quantidade de interações dos alunos com os cursos para gerar predições razoavelmente precisas, ainda que menos precisas do que as de trabalhos anteriores que utilizam atributos muito mais específicos. Entretanto, nos trabalhos já existentes a generalidade das aplicações para outros contextos é normalmente sacrificada pela especificidade dos atributos inseridos nos modelos, enquanto que a abordagem apresentada aqui é aplicável virtualmente em qualquer situação onde é possível contar interações de qualquer tipo.

Além disso, foi possível observar que os modelos apresentam uma baixa capacidade preditiva quando são utilizados apenas o número de interações dos estudantes e quando poucas semanas de dados estão disponíveis, mas que para ambos os cursos os modelos melhoram suas precisões à medida que os dados de novas semanas são inseridos.

Ainda, demonstrou-se que a derivação de atributos que levam em conta a quantidade de interações de professores e tutores, bem como outros atributos comparativos, e a utilização destes atributos nos modelos permite que os mesmos alcancem um desempenho bastante superior para as semanas iniciais em ambos os cursos testados. Esse resultado é particularmente importante, tendo em vista que a tarefa de predição da reprovação deve ocorrer em tempo hábil para que alguma medida possa ser tomada para evitar a concretização da reprovação ou evasão. É importante observar que estas derivações de atributos não violam o requisito de apenas utilizar contagens de interações genéricas, já que ainda não foram especificados em nenhum momento quais os tipos de interações estão sendo realizados.

Demonstrou-se também que as redes bayesianas se mostraram o modelo mais adequado para o problema e que é possível treinar o modelo utilizando diferentes fontes de exemplos. Em particular e para o Curso de Licenciatura em Educação do Campo, obtivemos precisões idênticas ao treinar tanto entre turmas, onde o treino é realizado sobre um conjunto de estudantes e a aplicação em outro conjunto disjunto, como entre semestres, onde o treino ocorre utilizando estudantes de um semestre e aplica-se em outro semestre.

Foi possível verificar que os modelos apresentam precisões diferentes entre cursos, indicando que dinâmicas diferentes influenciam na precisão da técnica. No entanto, a disponibilidade de apenas dois cursos não permite concluir sobre a capacidade de generalização da metodologia.
Diferentes trabalhos futuros são possíveis a partir dos resultados apresentados neste artigo. Uma questão importante é reduzir a diferença de desempenho dos modelos quando se utiliza apenas a contagem de interações e quando se utiliza atributos mais específicos. Para isso, parece ser necessário derivar outros atributos ou encontrar melhores modelos capazes de fazer uso dos mesmos. Adicionalmente, é necessário ampliar os testes aqui realizados para outros cursos, inclusive em outras instituições, de forma a melhor validar o modelo e sua capacidade de generalização. Testes da presente metodologia com bases de dados de outras instituições já foram iniciados por Queiroga et al. [24], mas ainda apresentando resultados muito preliminares.

Também interessante seria desmembrar as diferentes interações de acordo com os tipos relacionadas por Swan [17] e quantificar o quanto a inclusão dessas categorias (interações com o curso, interações entre pares, interações com professores e tutores) ajuda na melhora do desempenho dos modelos. Considerando que o grau de interatividade dos estudantes é também normalmente influenciado pela atuação dos professores e tutores envolvidos com o processo de ensino-aprendizagem [25], é possível que novos atributos derivados a partir das interações desses atores ajudem na melhoria da precisão dos modelos. Da mesma forma, experimentar com diferentes granularidades temporais (dias, semanas, meses etc.) para agrupar as contagens pode levar a um melhor desempenho, em particular em cursos muito ativos.

\section{Referências}

[1] ABED, CENSO EAD.BR: Relatório Analítico da Aprendizagem a Distância no Brasil. Curitiba: Associação Brasileira de Educação a Distância ABED, 2014.

[2] W. Hamalainen and M. Vinni, "Classifiers for educational data mining," in Handbook of educational data mining, C. Romero, S. Ventura, M. Pechenizkiy, and R. S. Baker, Eds., ed: CRC Press, 2011, pp. 57-71.

[3] T. Mishra, D. Kumar, and S. Gupta, "Mining Students' Data for Prediction Performance," in Advanced Computing \& Communication Technologies (ACCT), 2014 Fourth International Conference on, 2014, pp. 255-262.

[4] C. Romero and S. Ventura, "Educational Data Mining: A Review of the State of the Art," IEEE Transactions on Systems, Man, and Cybernetics, Part C, vol. 40, pp. 601-618, 2010. 
[5] M. Brown, "Learning Analytics: Moving from Concept to Practice," EDUCAUSE Learning Initiative Brief, 2012.

[6] L. P. Macfadyen and S. Dawson, "Mining LMS data to develop an "early warning system" for educators: A proof of concept," Computers \& Education, vol. 54, pp. 588-599, 2// 2010.

R. S. J. de Baker, S. Isotani, and A. M. J. B. de Carvalho, "Mineraçao de dados educacionais: Oportunidades para o brasil," Revista Brasileira de Informática na Educação, vol. 19, 2011.

D. Detoni, R. M. Araujo, and C. Cechinel, "Predição de Reprovação de Alunos de Educação a Distância Utilizando Contagem de Interações," in Anais do Simpósio Brasileiro de Informática na Educação, 2014, pp. 896-905.

[9] Á. F. Agudo-Peregrina, S. Iglesias-Pradas, M. Á. Conde-González, and Á. Hernández-García, "Can we predict success from log data in VLEs? Classification of interactions for learning analytics and their relation with performance in VLE-supported F2F and online learning," Computers in Human Behavior, vol. 31, pp. 542$550,2 / / 2014$.

[10] S. M. Jayaprakash, E. W. Moody, E. J. Lauría, J. R. Regan, and J. D. Baron, "Early Alert of Academically At-Risk Students: An Open Source Analytics Initiative," Journal of Learning Analytics, vol. 1, pp. 6-47, 2014.

[11] I. Lykourentzou, I. Giannoukos, V. Nikolopoulos, G. Mpardis, and V. Loumos, "Dropout prediction in e-learning courses through the combination of machine learning techniques," Computers \& Education, vol. 53, pp. 950-965, 11// 2009.

[12] V. C. Smith, A. Lange, and D. R. Huston, "Predictive Modeling to Forecast Student Outcomes and Drive Effective Interventions in Online Community College Courses," Journal of Asynchronous Learning Networks, vol. 16, pp. 51-61, 2012.

[13] S. Valsamidis, S. Kontogiannis, I. Kazanidis, and A. Karakos, "E-learning platform usage analysis," Interdisciplinary Journal of ELearning and Learning Objects, vol. 7, pp. 185204, 2011.

[14] M. Murray, J. Pérez, D. Geist, and A. Hedrick, "Student Interaction with Content in Online and Hybrid Courses: Leading Horses to the Proverbial Water," Informing Science, vol. 16, 2013.
[15]

W. P. Dickson, "Toward a deeper understanding of student performance in virtual high school courses: Using quantitative analyses and data visualization to inform decision making," $A$ synthesis of new research in $K-12$ online learning, pp. 21-23, 2005.

[16] D. R. Garrison, T. Anderson, and W. Archer, "Critical inquiry in a text-based environment: Computer conferencing in higher education," The internet and higher education, vol. 2, pp. 87-105, 1999.

[17] K. Swan, "Learning effectiveness online: What the research tells us," Elements of quality online education, practice and direction, vol. 4, pp. 1347, 2003.

[18] C. Beer, K. Clark, and D. Jones, "Indicators of engagement," presented at the Curriculum, technology \& transformation for an unknown future. Proceedings ascilite Sydney, 2010.

[19] L. M. B. Manhães, S. d. Cruz, R. J. M. Costa, J. Zavaleta, and G. Zimbrão, "Previsão de Estudantes com Risco de Evasão Utilizando Técnicas de Mineração de Dados," Anais do XXII SBIE-XVII WIE, Aracaju, 2011.

[20] E. Gottardo, C. Kaester, and R. V. Noronha, "Previsão de Desempenho de Estudantes em Cursos EAD Utilizando Mineração de Dados: uma Estratégia Baseada em Séries Temporais.," Anais do XXIII SBIE, 2012.

[21] R. L. Rodrigues, F. P. A. de Medeiros, and A. S. Gomes, "Modelo de Regressão Linear aplicador à previsão de desempenho de estudantes em ambiente de aprendizagem.," Anais do XXIV SBIE, 2013.

[22] E. Gottardo, C. Kaestner, and R. V. Noronha, "Previsão de Desempenho de Estudantes em Cursos EAD Utilizando Mineração de Dados: uma Estratégia Baseada em Séries Temporais," in Anais do Simpósio Brasileiro de Informática na Educação, 2012.

[23] M. Hall, E. Frank, G. Holmes, B. Pfahringer, P. Reutemann, and I. H. Witten, "The WEKA data mining software: an update," SIGKDD Explor. Newsl., vol. 11, pp. 10-18, 2009.

[24] E. Queiroga, C. Cechinel, and R. Araújo, "Um Estudo do Uso de Contagem de Interações Semanais para Predição Precoce de Evasão em Educação a Distância," Anais dos Workshops do Congresso Brasileiro de Informática na Educação, vol. 4, p. 1074, 2015. 
[25] C. Cechinel, "Quantitative Aspects about the Interactions of Professors in the Learning Management System during a Final Undergraduate Project Distance Discipline," Interdisciplinary Journal of E-Learning and Learning Objects (IJELLO), vol. 10, 2014. 